Journal of the Operations Research

Society of Japan

Vol. 38, No. 2, June 1995

\title{
A MEAN-VARIANCE-SKEWNESS PORTFOLIO OPTIMIZATION MODEL
}

\author{
Hiroshi Konno Ken-ichi Suzuki \\ Tokyo Institute of Technology
}

(Received May 10, 1993; Revised January 26, 1994)

\begin{abstract}
We will propose a mean-variance-skewness(MVS) portfolio optimization model, a direct extension of the classical mean-variance model to the situation where the skewness of the rate of return of assets and the third order derivative of a utility function play significant roles in choosing an optimal portfolio. The MVS model enables one to calculate an approximate mean-variance-skewness efficient surface, by which one can compute a portfolio with maximal expected utility for any decreasingly risk averse utility functions. Also, we propose three computational schemes for solving an associated nonconcave maximization problem, and some preliminary computational results will be reported.
\end{abstract}

\section{Introduction}

In [12], the authors proposed a mean-absolute deviation-skewness (MADS) portfolio optimization model, in which the lower semi-third moment of the rate of return of a portfolio is maximized subject to constraints on the mean and the absolute deviation of the rate of return. This model is an extension of the standard Mean-Variance (MV) model developed by $\mathrm{H}$. Markowitz [18], and is motivated by observations on the distribution of stock data in the market and on the practitioners' perception against risk.

The standard MV model is based upon the assumptions that an investor is risk averse and that either (i) the distribution of the rate of return is multi-variate normal, or (ii) the utility of the investor is a quadratic function of the rate of return. Unfortunately however, neither (i) nor (ii) holds in practice. It is now widely recognized that the real stock data do not follow a multi-variate normal distribution. To the contrary, detailed analysis of historical data observed in the Tokyo Stock Exchange (TSE) shows that the majority of them follow positively skewed distributions (See $[1,12,17]$ for details). Also, many investors prefer a positively skewed distributions to a negative one, if the expected value and variance are the same. Furthermore, some investors prefer a distribution with larger skewness at the expense of larger variance. This means that utility functions of investors are not quadratic. ${ }^{1}$

The importance of the third order moment in portfolio optimization has been suggested by P. Samuelson [24] in the late 50's. However, quantitative treatments of the third order moment were neglected until late 80 's due to several reasons. First it is very difficult to estimate the third order moment when $n$, the number of assets is over a few hundred. To set up an optimization model, we have to estimate ${ }_{n} C_{3} \simeq n^{3}$ correlation coefficients among three individual assets, in addition to ${ }_{n} C_{2} \simeq n^{2}$ covariance coefficients, which is very time consuming, if not impossible.

\footnotetext{
${ }^{1}$ In [17], Maghrebi tested the skewness preference and persistence hypothesis based on the extended CAPM which incorporate the effect of the third moment of rate of return using the data of TSE. He reported that the investors have a preference for positive skewness in their portfolios, and that it is not rejected that positively skewed assets in one period are likely to remain positively skewed in the next period. Similar results are also stated in [21].
} 
Second, the third order moment is not a concave function and hence it looked difficult to solve the resulting optimization problem by using standard computational methodologies. Let us note that it was not until late 80 's when a large scale mean-variance model became solvable on a real time basis.

Third, as shown by Merton [20], the mean-variance framework is sufficient (i.e., higher order moments can be ignored) if we allow continuous trading in the frictionless market. This assumption is obviously invalid in practice, but it provided a good reason for researchers to ignore the weary effects of higher order moments. Also, Kroll, Levy and Markowitz [15] states that the numerical comparison of the MV approach and the direct utility maximization approach using the historical data of up to 20 stocks led to almost the same result, so that the criticism against the MV model cannot be authorized. However their claim may not by valid when the number of stocks is over a few hundred. In fact, our computational studies [11] show that the behavior of a model with a small number of assets is often quite different from that with a large universe.

Meanwhile, the computational breakthrough of the last decade enabled one to solve a large scale MV model within a practical amount of time $[13,19,22,23,25]$. We are now able to draw an efficient frontier in the mean-variance plane very cheaply. These computational studies revealed several unexpected facts about the capital market. Of particular interest is that the market portfolio is located deep in the interior of the MV feasible set, contrary to the theoretical conclusion of the MV model. In fact, it sits so far from the mean-variance efficient frontier that it cannot be attributed to statistical errors or to the difference of the risk sensitivity of individual investors. We belive that this is another evidence that the MV model needs a modification to meet the reality of the capital market. Fortunately, the recent breakthrough in th computational aspects of financial optimization encourages us to pursue one step further. Several authors already tried to improve the MV model. Among them are the use of asymmetric risk functions [7,9], and axiomatic treatment of risk [8] as well as a direct utility maximization approach [16].

The purpose of this paper is to introduce a mean-variance-skewness (MVS) model, in which the third order moment of the rate of return is maximized subject to constraints on the mean and variance of the rate of return. By this, we can draw an efficient surface in the mean-variance-skewness space. The advantage of this approach is that it enables us to maximize the third order approximation of the expected utility for any decreasingly risk averse utility functions. This model is an outgrowth of an effort to relate the mean-absolute deviation-skewness (MADS) model developed earlier by the authors to the framework of expected utility maximization paradigm. In fact, MADS model can now be interpreted as one practical approximation scheme for solving an MVS model.

In Section 2, we will formulate the MVS model and relate it to the theory of expected utility maximization. Section 3 will be devoted to some discussions about the properties of utility functions and the statistical properties of the stock data. In section 4 and 5 , we will discuss computational schemes to solve the MVS model, and show some preliminary computational results of the MVS model using historical data in Section 6 .

\section{Utility Functions and the Distribution of Stock Data}

Let $R_{j}(j=1, \ldots, n)$ be a random variable representing the rate of return (per period) of the asset $S_{j}(j=1, \ldots, n)$. Also, let $x=\left(x_{1}, \cdots, x_{n}\right)$ be a portfolio where $x_{j}$ is the rate of investment into $S_{j}$. We will denote

$$
X=\left\{x \in \mathcal{R}^{n}: A x=b, x \geq 0\right\}
$$

where $A \in \mathcal{R}^{m \times n}, b \in \mathcal{R}^{m}$ and call $X$ an investable set. 
The rate of return $R(x)$ of a portfolio $x \in X$ is given by

$$
R(x)=\sum_{j=1}^{n} R_{j} x_{j}
$$

Let $U(x)$ be a utility of an investor associated with $x$. We will assume that $U$ is a function of $R(x)$, i.e.,

$$
U(x)=u(R(x))
$$

where $u(\cdot)$ satisfies the following assumption.

Assumption 1. $u(\cdot)$ is a decreasingly risk averse function, namely $u(\cdot)$ satisfies $u^{\prime}(\cdot)>0$, $u^{(2)}(\cdot)<0, u^{(3)}(\cdot)>0$ over the domain of $R(x), x \in X$.

Let $r_{j}$ be the expected value of $R_{j}$ and let $r(x)$ be the expected value of $R(x)$. Also, let

$$
\begin{aligned}
& r_{\min }=\min \{r(x): x \in X\} \\
& r_{\max }=\max \{r(x): x \in X\}
\end{aligned}
$$

Let us consider the Taylor's series expansion of $u(R(x))$ around the expected value $r(x)$ of $R(x)$ :

$$
u(R(x))=u(r(x))+\sum_{k=1}^{\infty} u^{(k)}(r(x))(R(x)-r(x))^{k} / k !
$$

Then we have

$$
E[u(R(x))]=u(r(x))+\sum_{k=1}^{\infty} u^{(k)}(r(x)) E\left[(R(x)-r(x))^{k} / k !\right]
$$

This expression leads us to select an optimal portfolio by means of the moment analysis. However, it is pointed out in [2] that the analysis based upon the first $l$ moments of the underlying distribution may lead to an erratic conclusion, i.e., the portfolio derived through this approach is not necessarily optimal from the viewpoint of expected utility maximization. In fact, if $l+1$ st order moment of the distribution is large compared to the first $l$ moments, then this approach is not valid. However we can safely use the first three moment approach since moment of order higher than four are negligible for the problems of our interest as will be discussed shortly. Further, let us note that, the validity of the MVS model is guaranteed if we assume the well known third-order stochastic dominance about the preference structure of investors [4].

Thus we will use the following third order approximation of the expected utility

$$
E[u(R(x))]=u(r(x))+u^{(2)}(r(x)) v(x) / 2+u^{(3)}(r(x)) k(x) / 6
$$

where

$$
\begin{aligned}
& v(x)=E\left[(R(x)-r(x))^{2}\right] \\
& k(x)=E\left[(R(x)-r(x))^{3}\right]
\end{aligned}
$$

In case the third term of the right hand side of (2.5) is small compared to the first and the second, maximal value of the expected utility can be obtained by solving

$$
\mid \begin{array}{ll}
\text { maximize } & u(r)+u^{(2)}(r) v(x) / 2 \\
\text { subject to } & r(x)=r, \quad x \in X
\end{array}
$$

for all achievable values of $r$. By Assumption $1, u^{(2)}(\cdot)<0$, so that this problem reduces to Markowitz's mean variance model:

$$
\mid \begin{array}{ll}
\operatorname{maximize} & v(x) \\
\text { subject to } & r(x)=r, \quad x \in X
\end{array}
$$


Let $x^{*}(r)$ and $v\left(x^{*}(r)\right)$ be an optimal solution and an optimal value of this quadratic programming problem, respectively. Then the best portfolio can be obtained by solving

$$
\mid \begin{array}{ll}
\text { maximize } & u(r)+u^{(2)}(r) v\left(x^{*}(r)\right) / 2 \\
\text { subject to } & r_{\min } \leq r \leq r_{\max }
\end{array}
$$

If, however, the third term of (2.5) is not negligible, we have to solve the following problem:

$$
\mid \begin{array}{ll}
\text { maximize } & u(r)+u^{(2)}(r) v(x) / 2+u^{(3)}(r) k(x) / 6 \\
\text { subject to } & r(x)=r, \quad x \in X
\end{array}
$$

We will argue in the sequel that the third order term of the Taylor's series expansion (2.5) is in fact not negligible, but that the higher order term is negligible.

First our previous work [12] revealed that the skewness

$$
K=k(R) /\{v(R)\}^{3 / 2}
$$

of the monthly rate of return $R$ of a majority of stocks in Tokyo Stock Exchange are positive. In fact, more than $60 \%$ of the 1118 stocks have large skewness which would reject the normality hypothesis at $5 \%$ significance level. Also, the average magnitude of skewness for 1118 stocks is 1.75 . Thus we find that the magnitude of $k(R)$ is about the same as $2\{v(R)\}^{3 / 2}$, which in turn implies roughly that the ratio $\alpha$ of the third order term over the second order term is given by

$$
\alpha \sim \frac{2 u^{(3)}(r) v^{\frac{1}{2}}(x)}{3 u^{(2)}(r)}
$$

Also, we know from [11] that the standard deviation of portfolios on the efficient frontier ranges from 0.02 to 0.06 . Thus if $u^{(3)}(r) / u^{(2)}(r)$ is greater than, say 5 , then the third order term would not be negligible.

Let us now consider the magnitude of

$$
\lambda(r)=u^{(3)}(r) / u^{(2)}(r)
$$

for three popular classes ${ }^{2}$ of risk averse utility functions [6]:

(a) HARA (hyperbolic absolute risk aversion)

$$
u(r)=(a r+b)^{c} \quad(a, b>0, c<1)
$$

(b) Negative Exponential

$$
u(r)=-e^{-a(1+r)} \quad(a>0)
$$

(c) Power

$$
u(r)=(1+r)^{c} \quad(c<1)
$$

Table 1 shows $\lambda(r)$ for each type of utility functions and a typical value of parameters for which the third order term is not negligible.

Also, it is easy to check that the third order derivatives $u^{(3)}(r)$ are positive for all three type of utility functions except when $1 \leq c \leq 2$ for the HARA class.

Next, let us check that the fourth order term is negligible compared to the second. According to our calculation, fourth order moments of the monthly rate of return of the NIKKEI 225 stocks range between 5 to 10 times $v^{2}$. Therefore the fourth order term is negligible if $u^{(4)}(r) / u^{(2)}(r)$ is less than 100 , which is the case for all utility functions with very rare exceptions.

\footnotetext{
${ }^{2}$ Note that the utility function of HARA (a) constitutes a very wide class and both (b) and (c) are special cases of (a).
} 


\begin{tabular}{|l|c|c|}
\hline utility function & $\lambda(r)$ & Not negligible when \\
\hline \hline HARA & $a(c-2) /(a r+b)$ & $b=1, a=1, c<-3$ \\
\hline Negative Exponential & $a$ & $a>5$ \\
\hline Power & $(2-c) /(1+r)$ & $c<-3$ \\
\hline
\end{tabular}

Table 1: $\lambda(r)$ of utility functions

\section{A Mean-Variance-Skewness Model}

Let us now consider the problem (2.11) in detail. We observed in the previous section that the third order derivatives of utility functions are usually positive, i.e., decreasingly risk averse.

Let us define the following maximization problem

$$
\mid \begin{array}{ll}
\operatorname{maximize} & k(x) \\
\text { subject to } & v(x)=s^{2} \\
& r(x)=r, \quad x \in X
\end{array}
$$

and let $x^{*}(r, s)$ and $f(r, s)$ be its optimal solution and optimal value, respectively and let

$$
F(r, s)=u(r)+u^{(2)}(r) s^{2} / 2+u^{(3)}(r) f(r, s) / 6
$$

Then the optimal solution of (2.11) will be obtained by finding the global maximum of $F(r, s)$ for all values of $(r, s)$ achievable over $X$. Note that if we have $f(r, s)$, then we would be able to solve (2.11) for any decreasingly risk averse class of utility functions.

Let

$$
\begin{aligned}
& q_{1}(r)=\min \{v(x): r(x)=r, x \in X\} \\
& q_{2}(r)=\max \{v(x): r(x)=r, x \in X\}
\end{aligned}
$$

Then the achievable set $S$ of $(r, s)$ is given by

$$
S=\left\{(r, s): q_{1}(r) \leq s^{2} \leq q_{2}(r), r_{\min } \leq r \leq r_{\max }, s \geq 0\right\}
$$

The lower boundary of S, i.e., the function of $q_{1}(r)$ is the so-called efficient frontier, which can be calculated by several algorithms $[13,19,25]$. Also, the upper boundary, $q_{2}(r)$ can be calculated by the enumerative algorithm developed by the authors [12].

The problem in the right hand side of $(3.1)$ is an non-concave maximization problem over a non-convex set. However, the non-convex constraint can be replaced by a convex constraint. To see this, let

$$
g(r, s)=\max \left\{k(x): r(x)=r, v(x) \leq s^{2}, x \in X\right\}
$$

and let $h^{2}(s)$ be the value of $v(x)$ corresponding to $g(r, s)$. Also, let

$$
G(r, s)=u(r)+u^{(2)}(r) h^{2}(s) / 2+u^{(3)}(r) g(r, s) / 6
$$

\section{Theorem 1.}

$$
\max \{G(r, s):(r, s) \in S\}=\max \{F(r, s):(r, s) \in S\}
$$




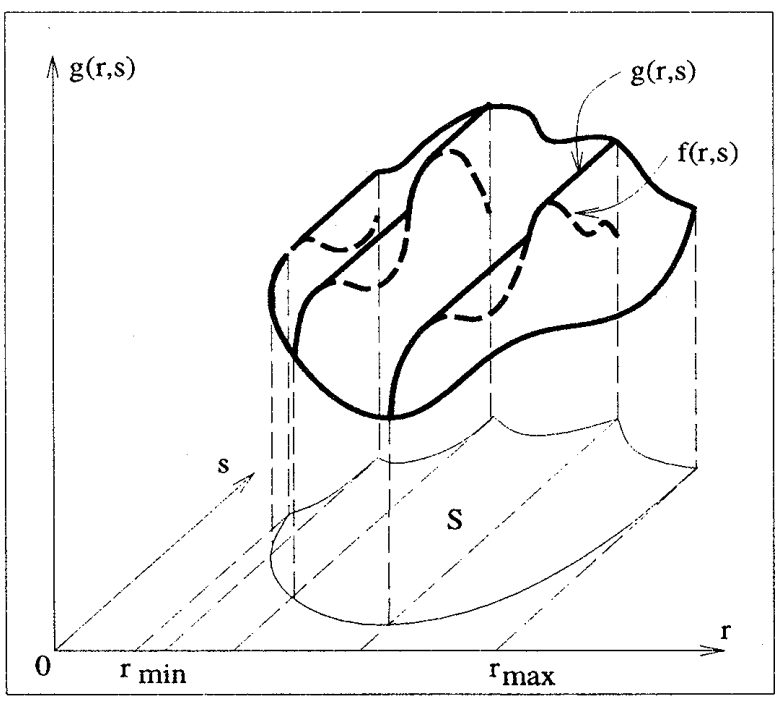

Figure 1: $f(r, s)$ and $g(r, s)$

\section{proof.}

By definition, $g(r, s) \geq f(r, s)$ for all $(r, s) \in S$. Also, since $u^{(2)}(r)<0$ for all $r \in\left[r_{\min }, r_{\max }\right]$, we have $G(r, s) \geq F(r, s)$ for all $(r, s) \in S$. Therefore

$$
\max \{G(r, s):(r, s) \in S\} \geq \max \{F(r, s):(r, s) \in S\}
$$

Let $\left(r^{*}, s^{*}\right)=\operatorname{argmax}\{G(r, s):(r, s) \in S\}$. Then it is easy to see that $g\left(r^{*}, s^{*}\right)=$ $f\left(r^{*}, h\left(s^{*}\right)\right)$, which in turn implies that

$$
G\left(r^{*}, s^{*}\right)=F\left(r^{*}, h\left(s^{*}\right)\right) .
$$

Relations (3.9) and (3.10) prove (3.8).

Figure 1 illustrates the behavior of $g(r, s)$. Also, Figure 2 shows the relationship between $f(r, s)$ and $g(r, s)$.

\section{Computational Schemes to Solve an MVS Model}

Let us now consider an algorithm for obtaining an approximate optimal solution of the problem.

$$
\begin{array}{|ll}
\text { maximize } & k(x) \\
\text { subject to } & v(x) \leq s^{2} \\
& r(x)=r, \quad x \in X
\end{array}
$$

We will assume that $\left(R_{1}, \cdots, R_{n}\right)$ is distributed over a discrete sample space and that

$$
f_{t}=\operatorname{Pr}\left\{\left(R_{1}, \cdots, R_{n}\right)=\left(r_{1 t}, \cdots, r_{n t}\right)\right\}, \quad t=1, \ldots, T
$$

is known. Then

$$
r_{j}=E\left[R_{j}\right]=\sum_{t=1}^{T} f_{t} r_{j t}
$$

Also,

$$
E[R(x)]=E\left[\sum_{j=1}^{n} R_{j} x_{j}\right]=\sum_{j=1}^{n} r_{j} x_{j}
$$




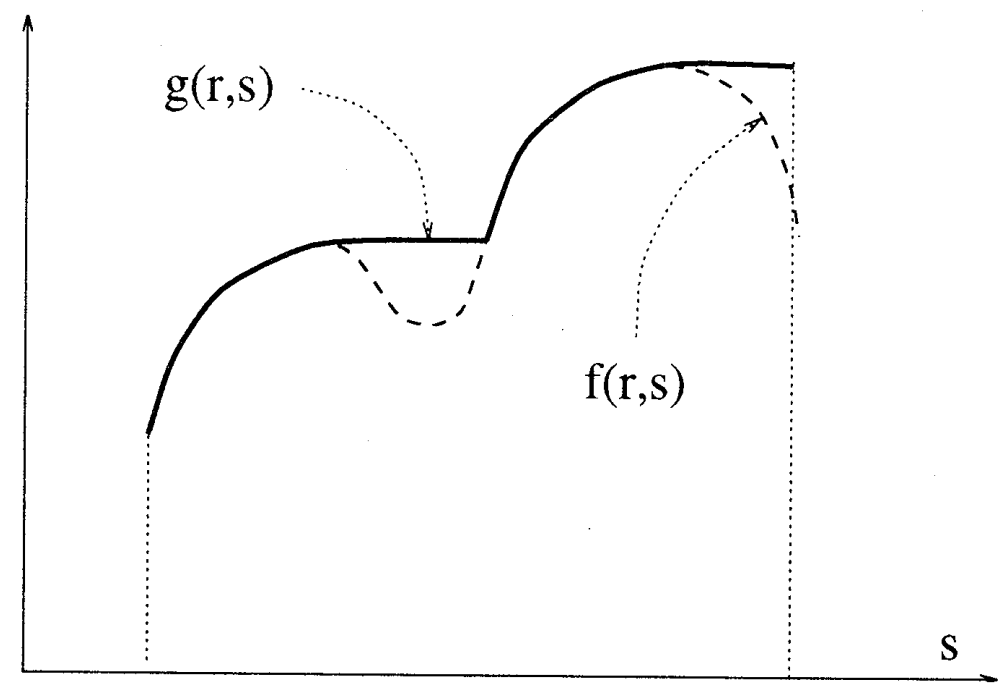

Figure 2: $f(r, s)$ and $g(r, s)$

$$
\begin{aligned}
V[R(x)] & =E\left[\left(\sum_{j=1}^{n}\left(R_{j}-r_{j}\right) x_{j}\right)^{2}\right] \\
& =E\left[\sum_{j=1}^{n} \sum_{k=1}^{n}\left(R_{j}-r_{j}\right)\left(R_{k}-r_{k}\right) x_{j} x_{k}\right] \\
& =\sum_{t=1}^{T} f_{t} \sum_{j=1}^{n} \sum_{k=1}^{n}\left(r_{j t}-r_{j}\right)\left(r_{k t}-r_{k}\right) x_{j} x_{k} \\
& =\sum_{t=1}^{T} f_{t}\left(\sum_{j=1}^{n}\left(r_{j t}-r_{j}\right) x_{j}\right)^{2}
\end{aligned}
$$

Similarly

$$
\begin{aligned}
k[R(x)] & =E\left[\left(\sum_{j=1}^{n}\left(R_{j}-r_{j}\right) x_{j}\right)^{3}\right] \\
& =E\left[\sum_{j=1}^{n} \sum_{k=1}^{n} \sum_{h=1}^{n}\left(R_{j}-r_{j}\right)\left(R_{k}-r_{k}\right)\left(R_{h}-r_{h}\right) x_{j} x_{k} x_{h}\right] \\
& =\sum_{t=1}^{T} f_{t} \sum_{j=1}^{n} \sum_{k=1}^{n} \sum_{h=1}^{n}\left(r_{j t}-r_{j}\right)\left(r_{k t}-r_{k}\right)\left(r_{h t}-r_{h}\right) x_{j} x_{k} x_{h} \\
& =\sum_{t=1}^{T} f_{t}\left(\sum_{j=1}^{n}\left(r_{j t}-r_{j}\right) x_{j}\right)^{3}
\end{aligned}
$$


Therefore the problem (4.1) can be written as follows:

$$
\begin{array}{|ll}
\text { maximize } & \sum_{t=1}^{T} f_{t} z_{t}^{3} \\
\text { subject to } & \sum_{t=1}^{T} f_{t} z_{t}^{2} \leq s^{2} \\
& \sum_{t=1}^{T} f_{t} z_{t}=r \\
& -z_{t}+\sum_{j=1}^{n} r_{j t} x_{j}=0, \quad t=1, \ldots, T, \quad x \in X
\end{array}
$$

(a) Piecewise Linear Approximation of the Quadratic Constraint In [11], we approximate $z_{t}^{2}$ by $\left|z_{t}\right| /(\pi / 2)^{\frac{1}{2}}$ by noting the relation

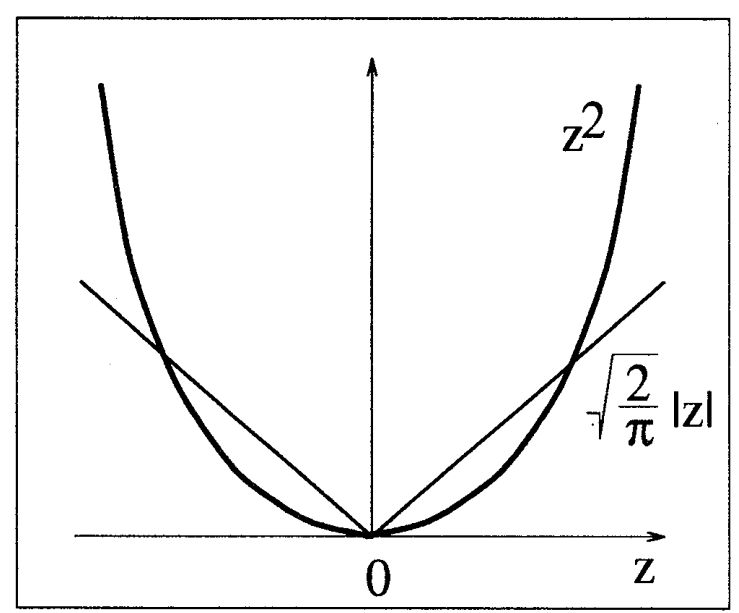

Figure 3-a

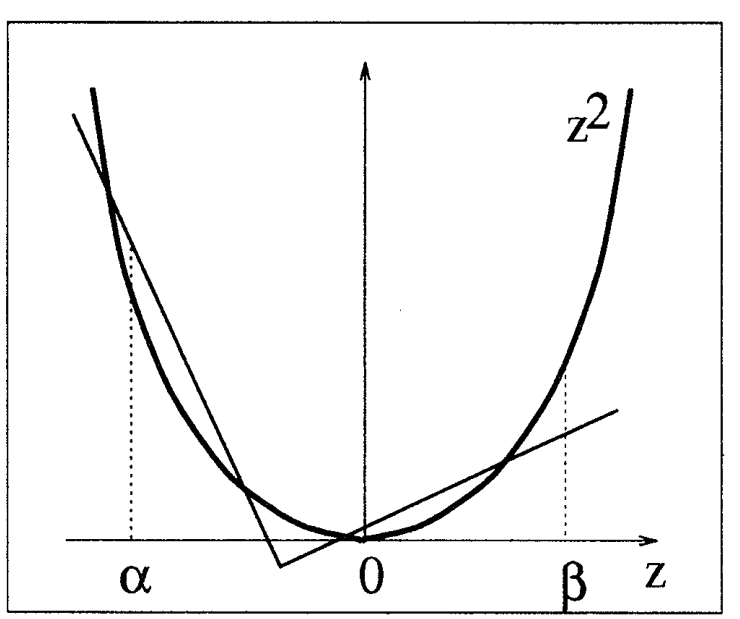

Figure $3-\mathrm{b}$

$$
\left\{E[|R-E[R]| \mid\}^{2}=\frac{2}{\pi} E\left[(R-E[R])^{2}\right]\right.
$$

when $R$ is normally distributed (Fig. 3-a).

Instead, we may employ a least square piecewise linear convex approximation (Fig. 3-b) over the interval $[\beta, \alpha]$ by solving a convex quadratic programing problem:

$$
\mid \begin{array}{ll}
\operatorname{minimize} & \int_{q}^{\alpha}\left(x^{2}-a_{1} x-b_{1}\right)^{2} d x+\int_{\beta}^{q}\left(x^{2}-a_{2} x-b_{2}\right)^{2} d x \\
\text { subject to } & a_{1} \geq a_{2} \\
& q_{1} q+b_{1}=a_{2} q+b_{2}
\end{array}
$$

where $q$ is usually taken as the mid-point of $\alpha$ and $\beta$. In particular, if $\alpha>0$ and $\beta=-\alpha$ then the optimal solution is given by

$$
a_{1}=-a_{2}=\alpha, \quad b_{1}=b_{2}=-\alpha^{2} / 6
$$

(b) Piecewise Linear Approximation of the Cubic Objective Function (i) 
In MADS model [11], we replaced the cubic function $z_{t}^{3}$ by a lower semi-cubic function (Fig. 4-a) and employed piecewise linear approximation. Though naive, this scheme successfully generated portfolio with large skewness.

To obtain a better approximation, we employ the least square piecewise linear convex approximation (Fig. 4-b.) by minimizing the following expressions.

$$
\mid \begin{array}{ll}
\operatorname{minimize} & \sum_{j=1}^{k} \int_{q_{j}}^{q_{j+1}}\left(x^{3}-a_{j} x-b_{j}\right)^{2} d x \\
\text { subject to } & a_{j} q_{j+1}+b_{j}=a_{j+1} q_{j+1}+b_{j+1}, \quad j=1, \ldots, k-1 \\
& a_{1} \geq a_{2} \cdots \geq a_{k}
\end{array}
$$

which is again a convex quadratic programming problem.

By these approximations, problem (4.7) is converted to a maximization of a piecewise linear concave function subject to piecewise linear convex constraints, which in turn can be converted to a linear programming problem $[3,5]$.

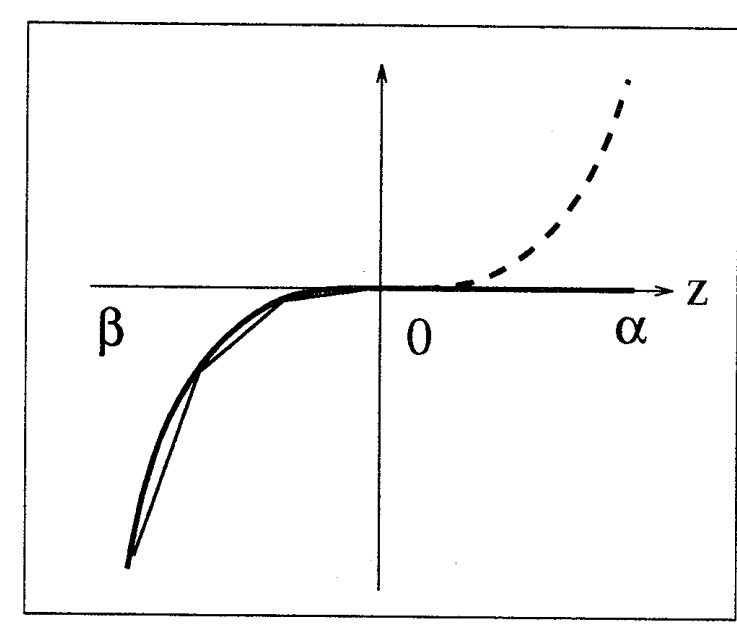

Figure 4-a

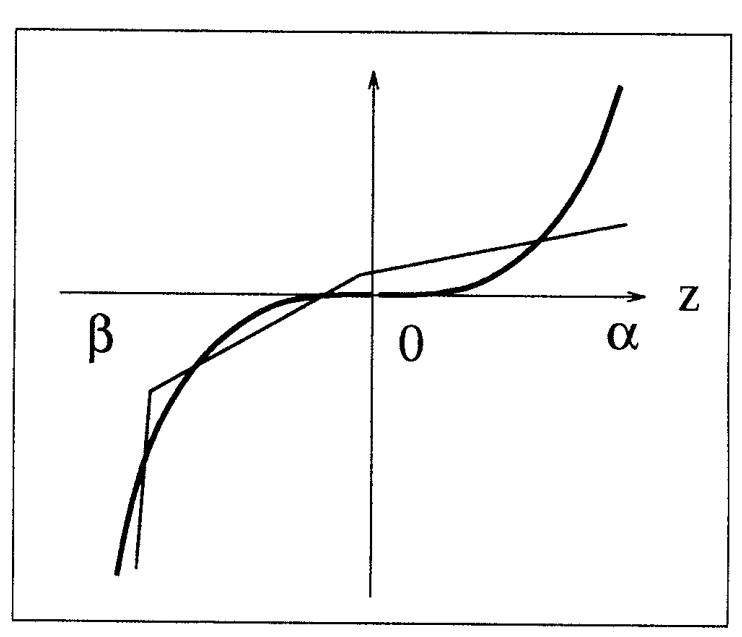

Figure 4-b

(c) Piecewise Linear Approximation of the Cubic Objective Function (ii)

The following is the another approximation of the objective function. Replacing the objective function by the sum of a lower semi-cubic function and an upper semi-cubic function, (4.7) can be written as follows:

$$
\mid \begin{array}{ll}
\text { maximize } & \sum_{t=1}^{T} f_{t}\left|z_{t}\right|_{-}^{3}+\sum_{t=1}^{T} f_{t}\left|z_{t}\right|_{+}^{3} \\
\text { subject to } & \left(z_{t}, x_{t}\right) \in Y
\end{array}
$$

where

$$
\begin{gathered}
Y=\left\{\left(z_{t}, x_{t}\right): \quad \sum_{t=1}^{T} f_{t} z_{t}^{2} \leq s^{2}, \sum_{t=1}^{T} f_{t} z_{t}=r\right. \\
\left.z_{t}-\sum_{j=1}^{n} r_{j t} x_{j}=0, \quad t=1, \ldots, T, \quad x \in X\right\} \\
|\xi|_{+}=\max \{0, \xi\}, \quad|\xi|_{-}=-\min \{0, \xi\} .
\end{gathered}
$$


By applying a standard technique, (4.10) can be converted to the following:

$$
\begin{array}{|ll}
\text { maximize } & \sum_{t=1}^{T} f_{t} w_{t}+\sum_{t=1}^{T} f_{t}\left|z_{t}\right|_{+}^{3} \\
\text { subject to } & w_{t}-\left|z_{t}\right|_{-\leq \leq \leq}^{\leq} 0, t=1, \ldots, T \\
& \left(z_{t}, x_{t}\right) \in Y, \quad t=1, \ldots, T .
\end{array}
$$

This problem is a maximization of a convex function over a convex set. Among the several possible methods, we employ a piecewise linear approximation (Fig. 5) and a mixed integer programming approach. In particular, we approximate the upper semi-cubic function $\left|z_{t}\right|_{+}^{3}$ by a piecewise linear convex function $\hat{f}\left(z_{t}\right)$ by introducing $K$ auxiliary variables $\lambda_{t k}$ :

$$
\left\{\begin{array}{l}
\hat{f}\left(z_{t}\right)=\sum_{k=1}^{K} \lambda_{t k}\left|\bar{z}_{t k}\right|_{+}^{3} \\
z_{t}=\sum_{k=1}^{K} \lambda_{t k} \bar{z}_{t k}, \quad t=1, \ldots, T \\
\sum_{k=1}^{K} \lambda_{t k}=1 \\
\lambda_{t k} \geq 0, \quad k=1, \ldots, K, \quad t=1, \ldots, T
\end{array}\right.
$$

where $\bar{z}_{t k}$ are $\mathrm{K}$ mesh points. We introduce $K-1 \quad 0-1$ variables corresponding to the mesh points and impose following constraints so that $z_{t}$ is expressed by a convex combination of at most two nearest mesh points ${ }^{3}$.

$$
\left\{\begin{array}{c}
\lambda_{t 1} \leq \delta_{t 1} \\
\lambda_{t 2} \leq \delta_{t 1}+\delta t 2 \\
\vdots \\
\lambda_{t K} \leq \delta_{t K-1} \\
\sum_{k=1}^{K-1} \delta_{t k}=1, \delta_{t k} \in[0,1]
\end{array}\right.
$$

\section{Direct Maximization of the Expected Utility}

If one is only interested in obtaining a portfolio with maximal expected utility for a given risk averse utility function, one can use the direct utility maximization approach below.

As before, let us assume that

$$
f_{t}=\operatorname{Pr}\left\{\left(R_{1}, \cdots, R_{n}\right)=\left(r_{1 t}, \cdots, r_{n t}\right)\right\}, \quad t=1, \ldots, T
$$

is known. Then the expected utility is represented as follows.

$$
E[u(R(x))]=\sum_{t=1}^{T} f_{t} u\left(\sum_{j=1}^{n} r_{j t} x_{j}\right)
$$

Therefore we have the following problem:

$$
\begin{array}{|ll}
\text { maximize } & \sum_{t=1}^{T} f_{t} u\left(y_{t}\right) \\
\text { subject to } & y_{t}-\sum_{j=1}^{n} r_{j t} x_{j}=0, t=1, \ldots, T \\
& x \in X
\end{array}
$$

\footnotetext{
${ }^{3}$ We can reduce the number of integer variable $\delta_{t k}$ by elimination of last equation of (4.13).
} 


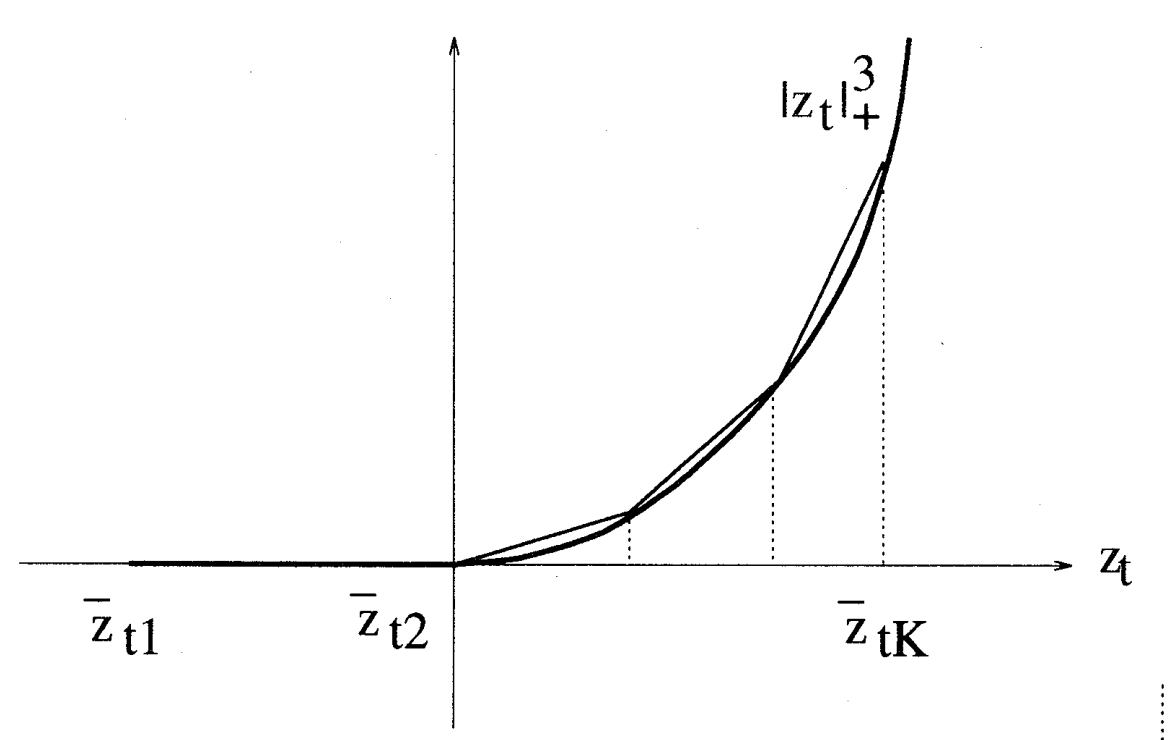

Figure 5: piecewise linear approximation

Since $u(\cdot)$ in concave, this problem can be solved by either one of the standard nonlinear programming algorithms or by the following piecewise linear approximation scheme.

Let us introduce auxiliary variables $\lambda_{t k}$ 's $(t=1, \ldots, T ; k=1, \ldots, K)$ which satisfy the following conditions

$$
\begin{aligned}
y_{t} & =\sum_{k=1}^{K} \lambda_{t k} u_{k} \\
\sum_{k=1}^{K} \lambda_{t k} & =1, \quad \lambda_{t k} \geq 0, \quad k=1, \ldots, K, \quad t=1 \ldots, T
\end{aligned}
$$

where $u_{k}=u\left(\xi_{k}\right), k=1, \ldots, K$.

The piecewise linear approximation of (5.3) is therefore

$$
\begin{array}{|ll}
\text { maximize } & \sum_{t=1}^{T} f_{t} \sum_{k=1}^{K} \lambda_{t k} u_{k} \\
\text { subject to } & \sum_{k=1}^{K} \lambda_{t k} \xi_{k}-\sum_{j=1}^{n} r_{j t} x_{j}=0, \quad t=1, \ldots, T \\
& \sum_{k=1}^{K} \lambda_{t k}=1, \lambda_{t k} \geq 0, k=1, \ldots, K, t=1, \ldots, T \\
& x \in X
\end{array}
$$

This is a linear programming problem with $n+T K$ variables and $T+K+1$ constraints and thus can be solved cheaply.

This is perhaps the easiest way to obtain a portfolio with maximal expected utility. However, it is not necessarily the best approach from the practitioner's point of view. In fact, it is not easy for a fund manager to specify an exact form of his utility function. In such cases, a lot of sensitivity analysis would have to be performed. The MVS model would be very helpful in such situations. 


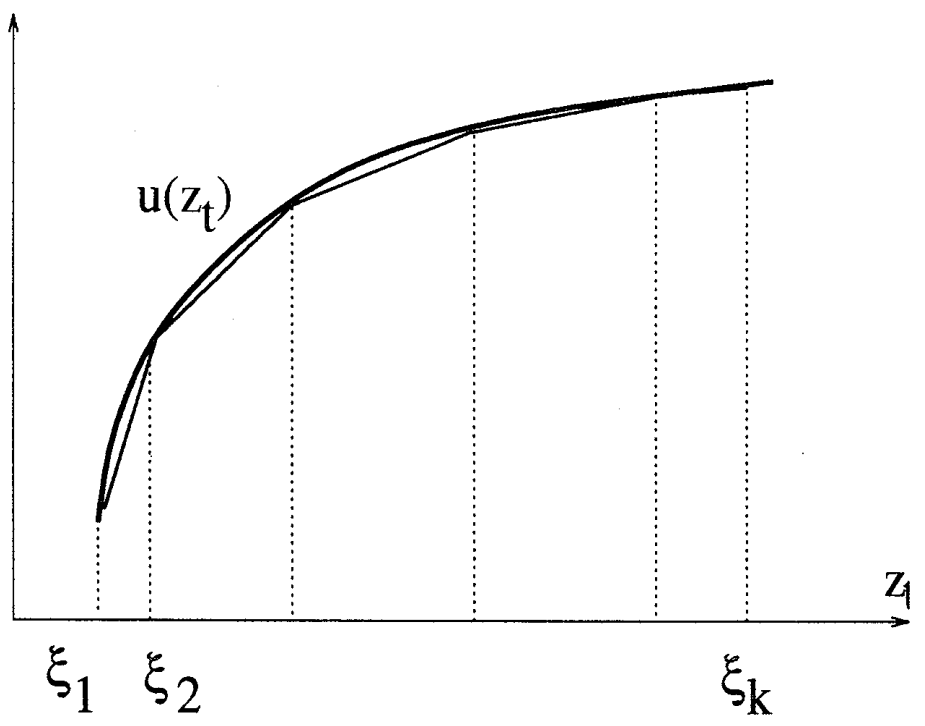

Figure 6

Let us point out that a utility maximization approach described above may be used to calculate the function $f(r, s)$ by solving the following problem:

$$
\begin{array}{|ll}
\text { maximize } & \sum_{t=1}^{T} f_{t} u\left(z_{t}\right) \\
\text { subject to } & \sum_{t=1}^{T} f_{t}\left(y_{t}-r\right)^{2}=s^{2} \\
& \sum_{t=1}^{T} f_{t} y_{t}=r \\
& y_{t}-\sum_{j=1}^{n} r_{j t} x_{j}=0, t=1, \ldots, T, x \in X
\end{array}
$$

Note that this problem is equivalent to

$$
\begin{array}{|ll}
\text { maximize } & E[u(R(x))] \\
\text { subject to } & V[R(x)]=s^{2} \\
& E[R(x)]=r, x \in X
\end{array}
$$

Hence the optimal value $v^{*}$ of this problem gives a third order approximation of $F(r, s)$ defined in (3.2). Therefore, we have

$$
f(r, s)=6\left\{u^{*}-u(r)-u^{(2)}(r) s^{2} / 2\right\} / u^{(3)}(r)
$$

where $x^{*}$ is an optimal solution of (5.6). Thus an exact optimal solution of (4.1) can be obtained by solving (5.6) for a cubic utility function which is decreasingly risk averse in the interval $\left[r_{\min }, r_{\max }\right]$.

Note however, that (5.6) is a concave maximization problem over a nonconvex set, for which an efficient algorithm has yet to be developed.

\section{Computational Experiments}

In this section, we will report some results of preliminary numerical experiments on the MVS model. We solved four problems using the same data set. One is the MAD model, 
and the remainder are three variants of the MVS model which we denote MVS1, MVS2 and MVS3, corresponding to section 3.-(a),3.-(b) and 3.-(c), respectively. We used three sets of historical data $D_{1}$ (from January ' 85 through December ' 86 ), $D_{2}$ (from January '86 through December '87) and $D_{3}$ (from January '89 through December '90) which consist of 24 monthly rate of return of the NIKKEI 225 stocks. The algorithm was coded in $\mathrm{C}$ language with the optimization library CPLEX which can handle both linear programs and the mixed integer programs, and was tested on SUN SPARCStation/1+. First we solved the MAD model at the rate of return $\rho$. Then we set the constraint on the standard deviation (s.d.) so that it does not exceed the level of 1.5 times of the s.d. calculated by using MAD model.

\begin{tabular}{|c|c|c|c|c|}
\hline \multicolumn{5}{|c|}{ D1('85/1-'86/12) } \\
\hline model & skewness & $\begin{array}{l}\text { Standard } \\
\text { deviation }\end{array}$ & $\dagger$ & CPU time(sec) \\
\hline MAD & 0.643 & $5.13 \mathrm{e}-3$ & & \\
\hline MVS1 & 0.315 & $7.70 \mathrm{e}-3$ & 17 & 4 \\
\hline MVS2 & 3.951 & $7.70 \mathrm{e}-3$ & 19 & 2 \\
\hline MVS3 & 6.988 & $7.70 \mathrm{e}-3$ & 20 & 31403 \\
\hline \multicolumn{5}{|c|}{$\mathrm{D} 2(187 / 1-' 88 / 12) \quad \rho: 4.0 \%$} \\
\hline MAD & 1.827 & $4.64 \mathrm{e}-3$ & & \\
\hline MVS1 & 6.734 & $6.97 \mathrm{e}-3$ & 19 & 2 \\
\hline MVS2 & -5.620 & $6.97 \mathrm{e}-3$ & 19 & 2 \\
\hline MVS3 & 9.412 & $6.97 \mathrm{e}-3$ & 18 & 18502 \\
\hline \multicolumn{5}{|c|}{$\overline{\mathrm{D} 3(' 89 / 1-' 90 / 12)} \quad \rho: 4.0 \%$} \\
\hline MAD & -38.284 & $60.2 \mathrm{e}-3$ & & \\
\hline MVS1 & 70.961 & $65.7 \mathrm{e}-3$ & 5 & 3 \\
\hline MVS2 & 109.904 & $90.3 \mathrm{e}-3$ & 7 & 2 \\
\hline MVS3 & 168.064 & 90.3 e-3 & 6 & 6618 \\
\hline
\end{tabular}

Table 2 shows the skewness, the standard deviation and the number of nonzero stocks of an optimal portfolio, as well as the CPU time for solving the problem. We see from this table that the MVS models generate a portfolio with larger skewness than MAD model in most cases, and that MVS3 model tends to achieve largest skewness. This is quite reasonable since the MVS3 model is the most precise approximation among the three. Unfortunately, however, it requires significantly more computational time compared with another three models because it is formulated as a mixed integer problem. In our experiments, we fixed the tolerance of MIP algorithm at $\epsilon=10^{-4}$, so that the optimal solution should be very exact. It is certainly possible to reduce computational time by somewhat relaxing this tolerance.

\section{Concluding Remarks}

We proposed an MVS portfolio optimization model which is a natural extension of the classical MV model to the situation where the third order term is not negligible.

One advantage of our approach is that it enables us to maximize the third order approximation of the expected utility for any decreasingly risk averse utility functions. It is not clear however, whether the MVS model is in fact better than MV model or MADS model. Also, we have to compare the relative advantage of computational schemes presented in Section 4 and 5. In any case, we have to conduct a lot of simulation to derive definite 
conclusions. More extensive numerical simulation is now under way, whose results will be reported subsequently.

\section{References}

[1] Aggarwal, R., Rao, R. P. and Hiraki, T.: "Skewness and Kurtosis in Japanese Equity Returns: Empirical Evidence", The J.of Financial Research, Vol. 12 (1989), 253-260.

[2] Brockett, P. and Y.Kahane, : "Risk, Return, Skewness and Preference", Management Science, Vol. 38 (1992), 851-866.

[3] Chvátal, V.: Linear Programming, Freeman and Co., New York, 1983.

[4] Elton, E. and M.J.Gruber, : Modern Portfolio Theory and Investment Analysis (4th edition), John Wiley and Sons, Inc, 1991.

[5] Hillier, F. and G.J.Lieberman, : Introduction to Operations Research (3rd edition), Holden-Day, Inc., 1984.

[6] Ingersoll Jr., J.: Theory of Financial Decision Making, Rowman \& Littlefield, 1987.

[7] Jensen, D. and A.J.King, : "Linear-Quadratic Efficient Frontiers for Portfolio Optimization", Applied Stochastic Models and Data Analysis, Vol. 8 (1992), 195-207.

[8] Kijima, M. and M.Ohnishi, : "Mean-Risk Analysis of Risk Aversion and Wealth Effects on Optimal Portfolio with Multiple Investment ", Annals of Operations Research, Vol. 45 (1993).

[9] King, A.: " Asymmetric Risk Measures and Tracking Models for Portfolio Optimization under Uncertainty", Annals of Operations Research, Vol. 45 (1993).

[10] Konno, H.: "Piecewise Linear Risk Functions and Portfolio Optimization ", J.of the Operations Research Society of Japan, Vol. 33 (1990), 139-156.

[11] Konno, H. and H.Yamazaki,: "A Mean-Absolute Deviation Portfolio Optimization Model and Its Applications to Tokyo Stock Market", Management Science, Vol. 37 (1991), 519-531.

[12] Konno, H. and H.Yamazaki, : "A Mean-Absolute Deviation -Skewness Portfolio Optimization Model", Annals of Operations Research, Vol. 45 (1993).

[13] Konno, H. and K.Suzuki, : "A Fast Algorithm for Solving Large Scale Mean-Variance Models by Compact Factorization of Covariance Matrices", J.of the Operations Research Society of Japan, Vol. 35 (1992), 93-104.

[14] Konno, H., S.Pliska, and K.Suzuki, : "Optimal Portfolio with Asymptotic Criteria", Annals of Operations Research, Vol. 45 (1993).

[15] Kroll, Y., H.Levy, and H.M.Markowitz, : "Mean-Variance versus Direct Utility Maximization ", J. of Finance, Vol. 39 (1984), 47-62.

[16] Levy, H. and H.M.Markowitz, : "Approximating Expected Utility by a Function of Mean and Variance", American Economic Review, (1979), 308--317.

[17] Maghrebi, N.: "On Skewness Preference and Persistence Hypotheses ", Japan Financial Review, Vol. 15 (1992), 17-35.

[18] Markowitz, H.: Portfolio Selection: Efficient Diversification of Investments, John Wiley and Sons, Inc, 1959.

[19] Markowitz, H., et al.: "Fast Computation of Mean-Variance Efficient Sets Using Historical Variance", Technical report, Daiwa Securities America, 1991, (To appear in J.of Financial Engineering).

[20] Merton, R.: "Optimum Consumption and Portfolio Rules in Continuous-Time Model", J.of Economic Theory, Vol. 3 (1971), 373-413.

[21] Muralidhar, K.: "The Bootstrap Approach for Testing Skewness Persistence ", Management Science, Vol. 39 (1993), 487-491.

[22] Nakasato, M.: "Some Characteristics of the Efficient Frontier for TSE Stocks ", Technical report, Dept.of IE \& Management, Tokyo Institute of Technology, 1991. 
[23] Perold, A.: "Large Scale Portfolio Optimization", Management Science, Vol. 30 (1984), 1143-1160.

[24] Samuelson, P.: "The Fundamental Approximation Theorem of Portfolio Analysis in Terms of Means Variances and Higher Moments", Review of Economic Studies, Vol. 25 (1958), 65-86.

[25] Takehara, H.: "An Interior Point Algorithm for Large Scale Portfolio Optimization", Annals of Operations Research, Vol. 45 (1993).

Hiroshi KONNO, Ken-ichi SUZUKI:

Industrial Engineering and Management, Tokyo Institute of Technology, 2-12-1 Ohokayama, Megurho-ku, Tokyo 152, Japan e-mail kenichi@me.titech.ac.jp 